\title{
Constant-Round Black-Box Construction of Composable Multi-Party Computation Protocol
}

\author{
Susumu Kiyoshima ${ }^{1}$, Yoshifumi Manabe ${ }^{2}$, and Tatsuaki Okamoto ${ }^{1}$ \\ 1 NTT Secure Platform Laboratories, Japan \\ \{kiyoshima. susumu, okamoto.tatsuaki\}@lab.ntt.co.jp \\ ${ }^{2}$ Kogakuin University, Japan \\ manabe@cc.kogakuin.ac.jp
}

\begin{abstract}
We present the first general MPC protocol that satisfies the following: (1) the construction is black-box, (2) the protocol is universally composable in the plain model, and (3) the number of rounds is constant. The security of our protocol is proven in angel-based UC security under the assumption of the existence of one-way functions that are secure against sub-exponential-time adversaries and constant-round semi-honest oblivious transfer protocols that are secure against quasi-polynomial-time adversaries. We obtain the MPC protocol by constructing a constant-round CCA-secure commitment scheme in a black-box way under the assumption of the existence of one-way functions that are secure against subexponential-time adversaries. To justify the use of such a sub-exponential hardness assumption in obtaining our constant-round CCA-secure commitment scheme, we show that if black-box reductions are used, there does not exist any constant-round CCA-secure commitment scheme under any falsifiable polynomial-time hardness assumptions.
\end{abstract}

\section{Introduction}

Protocols for secure multi-party computation (MPC) enable mutually distrustful parties to compute a functionality without compromising the correctness of the outputs and the privacy of the inputs. In the seminal work of Goldreich et al. 14, a general MPC protocol was constructed in a model with malicious adversaries and a dishonest majority 1 (By "a general MPC protocol," we mean a protocol that can be used to securely compute any functionality.)

Black-box Constructions. A construction of a protocol is black-box if it uses the underlying cryptographic primitives only in a black-box way (that is, only through their input/output interfaces). In contrast, if a construction uses the codes of the underlying primitives, it is non-black-box.

Obtaining black-box constructions is an important step toward obtaining practical MPC protocols. This is because black-box constructions are typically more efficient than non-black-box ones. (Typical non-black-box constructions,

\footnotetext{
${ }^{1}$ In the following, we consider only such a model. 
such as that of [14, use the codes of the primitives to compute NP reductions in general zero-knowledge proofs. Thus, they should be viewed as feasibility results.) Black-box constructions are also theoretically interesting, since understanding whether non-black-box use of primitives is necessary for a cryptographic task is of great theoretical interest.

Recently, a series of works showed black-box constructions of general MPC protocols. Ishai et al. [20] showed the first construction of a general MPC protocol that uses the underlying low-level primitives in a black-box way. Combined with the subsequent work of Haitner [18, their work showed a black-box construction of a general MPC protocol based on a semi-honest oblivious transfer protocol [19. Subsequently, Wee 37] showed an $O\left(\log ^{*} n\right)$-round protocol under polynomial-time hardness assumptions and a constant-round protocol under sub-exponential-time hardness assumptions, and Goyal [15] showed a constantround protocol under polynomial-time hardness assumptions.

The security of these black-box protocols is considered in the stand-alone setting. That is, the protocols of [15,20,37] are secure in the setting where only a single instance of the protocol is executed at a time.

Composable Security. The concurrent setting, in which many instances of protocols are executed concurrently in an arbitrary schedule, is a more general and realistic setting than the stand-alone one. In the concurrent setting, an adversary can perform a coordinated attack in which he chooses his messages in an instance based on the executions of the other instances.

As a strong and realistic security notion in the concurrent setting, Canetti [2] proposed universally composable $(U C)$ security. The main advantage of UC security is composability, which guarantees that when we compose many UCsecure protocols, we can prove the security of the resultant protocol using the security of its components. Thus, UC security enables us to construct protocols in a modular way. Composability also guarantees that a protocol remains secure even when it is concurrently executed with any other protocols in any schedule. Canetti et al. 8] constructed a UC-secure general MPC protocol in the common reference string $(C R S)$ model (i.e., in a model in which all parties are given a common public string that is chosen by a trusted third party).

UC security, however, turned out to be too strong to achieve in the plain model (i.e., in a model without any trusted setup except for authenticated communication channels). That is, we cannot construct UC-secure general MPC protocols in the plain model [3, 6].

To achieve composable security in the plain model, Prabhakaran and Sahai [36] proposed a variant of UC security called angel-based UC security. Roughly speaking, angel-based UC security is the same as UC security except that the adversary and the simulator have access to an additional entity - the angelthat allows some judicious use of super-polynomial-time resources. It was proven that, like UC security, angel-based UC security guarantees composability. Furthermore, as argued in [36], angel-based UC security guarantees meaningful 
security in many cases. (For example, angel-based UC security implies superpolynomial-time simulation (SPS) security 1, 12, 29, 31. In SPS security, we allow the simulator to run in super-polynomial time. Thus, SPS security guarantees that whatever an adversary can do in the real world can also be done in the ideal world in super-polynomial time.) Then, Prabhakaran and Sahai 36] presented a general MPC protocol that satisfies this security notion in the plain model, based on new (unstudied and non-standard) assumptions. Subsequently, Malkin et al. 25] constructed another general MPC protocol that satisfies this security notion in the plain model based on new number-theoretic assumption. In [1], Barak and Sahai remarked that their protocol (which is SPS secure under subexponential-time hardness assumptions) can be shown to be secure in angel-based UC security.

Recently, Canetti et al. constructed a polynomial-round general MPC protocol in angel-based UC security based on a standard assumption (the existence of enhanced trapdoor permutations). Subsequently, Lin [21] and Goyal et al. [17. reduced the round complexity to $\widetilde{O}(\log n)$ under the same assumption. They also proposed constant-round protocols, where the security is based on a super-polynomial-time hardness assumption (the existence of enhanced trapdoor permutations that are secure against quasi-polynomial-time adversaries). These constructions, however, use the underlying primitives in a non-black-box way.

Black-Box Constructions of Composable Protocols. Lin and Pass 23$]$ showed the first black-box construction of a general MPC protocol that guarantees composable security in the plain model. The security of their protocol is proven under angel-based UC security, and based on the minimum assumption of the existence of semi-honest oblivious transfer (OT) protocols.

The round complexity of their protocol is $O\left(n^{\epsilon}\right)$, where $\epsilon>0$ is an arbitrary constant. In contrast, for non-black-box constructions of composable protocols, we have constant-round protocols in the plain model (under non-standard assumptions or super-polynomial-time hardness assumptions) [17,21,25,36]. Thus, a natural question is the following.

Does there exist a constant-round black-box construction of a general $M P C$ protocol that guarantees composability in the plain model (possibly under super-polynomial-time hardness assumptions)?

\subsection{Our Result}

In this paper, we answer the above question affirmatively.

Theorem (Informal). Assume the existence of one-way functions that are secure against sub-exponential-time adversaries and constant-round semi-honest oblivious transfer protocols that are secure against quasi-polynomial-time adversaries. Then, there exists a constant-round black-box construction of a general $M P C$ protocol that satisfies angel-based UC security in the plain model.

The formal statement of this theorem is given in Section 7. 
CCA-Secure Commitment Schemes. We prove the above theorem by constructing a constant-round CCA-secure commitment scheme [7,23] in a blackbox way. Once we obtain a CCA-secure commitment scheme, we can construct a general MPC protocol in essentially the same way as Lin and Pass do in 23.

Roughly speaking, a CCA-secure commitment scheme is a tag-based commitment scheme (i.e., a commitment scheme that takes an $n$-bit string, or tag, as an additional input) such that the committed value of a commitment with tag id remains hidden even if the receiver has access to a super-polynomial-time oracle - the committed-value oracle - that returns the committed value of any commitment with tag $\mathrm{id}^{\prime} \neq$ id. Lin and Pass 23 . showed an $O\left(n^{\epsilon}\right)$-round blackbox construction of a CCA-secure commitment scheme for arbitrary $\epsilon>0$ by assuming the minimum assumption of the existence of one-way functions.

Our main technical result is the following.

Theorem (Informal). Assume the existence of one-way functions that are secure against sub-exponential-time adversaries. Then, there exists a constantround black-box construction of a CCA-secure commitment scheme.

The formal statement of this theorem is given in Section 7.

To obtain our CCA-secure commitment scheme, we use the idea of nonmalleability amplification that was used in previous works on concurrent nonmalleable (NM) commitment schemes [22, 34. That is, we construct a CCA commitment scheme in the following steps.

Step 1. We say that a commitment scheme is one-one CCA secure if it is CCA secure with respect to restricted classes of adversaries that receive only a single answer from the oracle. Then, we construct a constant-round one-one CCA-secure commitment for tags of length $O(\log \log \log n)$.

Step 2. We construct a transformation from the commitment scheme constructed in Step 1 to a CCA-secure commitment for tags of length $O(n)$ with a constant additive increase in round complexity. Toward this end, we construct the following two transformations:

- A transformation from any one-one CCA-secure commitment scheme for tags of length $t(n)$ to a CCA-secure commitment scheme for tags of length $t(n)$ with a constant additive increase in round complexity

- A transformation from any CCA-secure commitment scheme for tags of length $t(n)$ to a one-one CCA-secure commitment scheme for tags of length $2^{t(n)-1}$ with no increase in round complexity

(The latter transformation is essentially the same as the "DDN $\log n$ trick" $[11,24$.) By repeatedly composing these two transformations, we obtain the desired transformation.

On the Use of Super-Polynomial-Time Hardness Assumption. Although the round complexity of our CCA-secure commitment scheme is constant, it relies on a super-polynomial-time hardness assumption. (Recall that the $O\left(n^{\epsilon}\right)$-round CCA-secure commitment scheme of [23] relies on a polynomial-time hardness assumption.) 
We show that the use of such a strong assumption is inevitable, as long as the security of a constant-round CCA-secure commitment scheme is proven under falsifiable assumptions [13, 28, by using a black-box reduction. Roughly speaking, a falsifiable assumption is an assumption that is modeled as an interactive game between a challenger and an adversary such that the challenger can decide whether the adversary won the game in polynomial time. Then, we say that the $C C A$ security of a commitment scheme $\langle C, R\rangle$ is proven under a falsifiable assumption by using a black-box reduction if the CCA security of $\langle C, R\rangle$ is proven by constructing a PPT Turing machine $\mathcal{R}$ such that for any adversary $\mathcal{A}$ that breaks the CCA security of $\langle C, R\rangle, \mathcal{R}$ can break the assumption by using $\mathcal{A}$ only in a black-box way. Then, we show the following theorem.

Theorem (Informal). Let $\langle C, R\rangle$ be any constant-round commitment scheme. Then, the $C C A$ security of $\langle C, R\rangle$ cannot be proven by using black-box reductions under any falsifiable polynomial-time hardness assumption.

(Due to lack of space, we defer the formal statement of this theorem and its proof to the full version. Roughly speaking, we obtain this theorem by using techniques of the negative result on concurrent zero-knowledge protocols [5.) Since all standard cryptographic assumptions are falsifiable, this theorem says that if we want to construct a constant-round CCA-secure commitment scheme based on standard assumptions, we must use either super-polynomial-time hardness assumptions (as this paper does) or non-black-box reductions 2

We note that this negative result holds even for non-black-box constructions. That is, we cannot construct constant-round CCA-secure commitment schemes even when we use primitives in a non-black-box way, as long as we use black-box reductions and polynomial-time hardness assumptions.

\section{Overview of the Protocols}

In this section, we give overviews of our main technical results: a one-one CCAsecure commitment scheme for short tags and a transformation from one-one CCA security to CCA security.

\subsection{One-One CCA-Security for Short Tags}

We obtain our one-one CCA-secure commitment scheme by observing that the non-black-box construction of a NM commitment scheme of 34] is one-one CCA secure and converting it into a black-box one.

First, we recall the scheme of [34] 3 The starting point of the scheme is "twoslot message length" technique [30. The basic idea of the technique is to let the receiver sequentially send two challenges - one "long" and one "short" - where

\footnotetext{
${ }^{2}$ We note that, although very recently Goyal 16 showed how to use non-black-box techniques in the fully concurrent setting, Goyal's technique requires polynomially many rounds.

${ }^{3}$ In the following, some of the text is taken from 34.
} 
the length of the challenges are determined by the tag of the commitment. The protocol is designed so that the response to a shorter challenge does not help a man-in-the-middle adversary to provide a response to a longer challenge. A key conceptual insight of [34] is to rely on the complexity leveraging technique [4] to construct these challenges: For one-way functions with sub-exponential hardness, an oracle for inverting challenges of length $n^{o(1)}$ (the "short" challenge) does not help invert random challenges of length $n$ (the "long" challenge), since we can simulate such an oracle by brute force in time $2^{n^{o(1)}}$.

More precisely, the scheme of [34] is as follows. Let $d=O(\log \log n)$ be the number of tags, and let $n^{\omega(1)}=T_{0}(n) \ll T_{1}(n) \ll \cdots \ll T_{d+2}(n)$ be a hierarchy of running times. Then, to commit to $v \in\{0,1\}^{n}$ with tag id $\in\{0,1, \ldots, d-1\}$, the committer $C$ does the following with the receiver $R$.

1. $C$ commits to $v$ by using a statistically binding commitment Com that is hiding against $T_{d+1}(n)$-time adversaries but is completely broken in time $T_{d+2}(n)$.

2. (Slot 1) $C$ proves knowledge of $v$ by using a zero-knowledge argument of knowledge that is computationally sound against $T_{\mathrm{id}+1}(n)$-time adversaries and can be simulated in straight line in time $o\left(T_{\mathrm{id}+2}(n)\right)$, where the simulated view is indistinguishable from the real one in time $T_{d+2}(n)$.

3. (Slot 2) $C$ proves knowledge of $v$ by using a zero-knowledge argument of knowledge that is computationally sound against $T_{d \text {-id }}(n)$-time adversaries and can be simulated in straight line in time $o\left(T_{d-\mathrm{id}+1}(n)\right)$, where the simulated view is indistinguishable from the real one in time $T_{d+2}(n)$.

We can show that the scheme of 34 is one-one CCA secure as follows (by using essentially the same proof as the proof of its non-malleability). Recall that a commitment scheme is one-one CCA secure if it is hiding against adversaries that give a single query to the committed-value oracle $\mathcal{O}$. Let id be the tag used in the left session (a commitment from the committer to the adversary $\mathcal{A}$ ) and $\widetilde{\text { id }}$ be the tag used in the right session (a commitment from $\mathcal{A}$ to $\mathcal{O}$ ). Then, let us consider a hybrid experiment in which the proofs in the second and third steps are replaced with the straight-line simulations in the left session. Since the running time of $\mathcal{O}$ is at most $T_{d+2}(n)$, the zero-knowledge property guarantees that the view of $\mathcal{A}$ in the hybrid experiment is indistinguishable from that of $\mathcal{A}$ in the real experiment even when $\mathcal{A}$ interacts with $\mathcal{O}$. Furthermore, in the right session of the hybrid experiment, the soundness of the zero-knowledge argument still holds either in the second step or in the third step. This follows from the following reasons. For simplicity, let us consider a synchronized adversary 4 Then, since the simulation of the second step takes at most time $o\left(T_{\mathrm{id}+2}(n)\right)$ and the soundness of the second step holds against $T_{\widetilde{\mathrm{id}}+1}(n)$-time adversaries, the soundness of the second step holds if id $<\widetilde{i d}$; similarly, the soundness of the third step holds if id $>\widetilde{i d}$. In the hybrid experiment, therefore, the committed value $v$ can be extracted by using the knowledge extractor either in the

${ }^{4}$ An synchronized adversary sends the $i$-th round message to $\mathcal{O}$ immediately after receiving the $i$-th round messages from the committer, and vise verse. 
second step or in the third step, and thus the committed value oracle $\mathcal{O}$ can be simulated in time $o\left(\max \left(T_{\mathrm{id}+2}(n), T_{d-\mathrm{id}+1}(n)\right)\right) \cdot \operatorname{poly}(n) \ll T_{d+1}(n)$. Then, from the hiding property of Com in the first step, the view of $\mathcal{A}$ in the hybrid experiment is computationally independent of the value $v$. Thus, one-one CCA security follows.

To convert the scheme of [34] into a black-box protocol, we use a black-box trapdoor commitment scheme TrapCom of 33 . We observe that TrapCom has similar properties to the zero-knowledge argument used in the scheme of [34]: TrapCom is extractable and a TrapCom commitment can be simulated in straight line in super-polynomial time. Then, we modify the scheme of [34] and let the committer commit to $v$ instead of proving the knowledge of $v$. To ensure the "soundness," that is, to ensure that the committed value of TrapCom is $v$, we use the cut-and-choose technique and Shamir's secret sharing scheme in a similar manner to previous works on black-box protocols $9,10,23,37$. That is, we let the committer commit to Shamir's secret sharing $s=\left(s_{1}, \ldots, s_{10 n}\right)$ of value $v$ in all steps, let the receiver choose a random subset $\Gamma \subset[10 n]$ of size $n$, and let the committer reveal $s_{j}$ and decommit the corresponding commitments for every $j \in \Gamma$. The resultant scheme uses the underlying primitives only in a black-box way, and can be proven to be one-one CCA secure from a similar argument to the scheme of [34. (We note that the actual scheme is a little more complicated. For details, see Section 4.) We note that Lin and Pass 23 also use TrapCom to convert a non-black-box protocol into a black-box one. Unlike them, who mainly use the fact that TrapCom is extractable and is secure against selective opening attacks, we also use the fact that TrapCom commitments are straight-line simulatable.

\subsection{CCA Security from One-One CCA Security}

We give an overview of the transformation from any one-one CCA-secure commitment scheme to a CCA-secure commitment scheme. Let $n^{\omega(1)}=T_{0}(n) \ll$ $T_{1}(n) \ll T_{2}(n) \ll T_{3}(n)$ be a hierarchy of running times. Then, we construct a CCA-secure commitment scheme CCACom $_{0}$ that is secure against $T_{0}(n)$-time adversaries from a one-one CCA-secure commitment scheme CCACom $_{3}^{1: 1}$ that is secure against $T_{3}(n)$-time adversaries. Let $\operatorname{Com}_{1}$ be a 2-round statistically binding commitment scheme that is secure against $T_{1}(n)$-time adversaries but is completely broken in time $o\left(T_{2}(n)\right)$, and CECom 2 be a constant-round commitment scheme that is hiding against $T_{2}(n)$-time adversaries and is concurrently extractable by rewinding the committer poly $\left(n^{\log n}\right)$ times 26,32 . Then, to commit to value $v$, the committer $C$ does the following with the receiver $R$.

1. $R$ commits to a random subset $\Gamma \subset[10 n]$ of size $n$ by using CCACom $_{3}^{1: 1}$.

2. $C$ computes an $(n+1)$-out-of- $10 n$ Shamir's secret sharing $\boldsymbol{s}=\left(s_{1}, \ldots, s_{10 n}\right)$ of value $v$ and commits to $s_{j}$ for each $j \in[10 n]$ in parallel by using Com $_{1}$.

3. $C$ commits to $s_{j}$ for each $j \in[10 n]$ in parallel by using CECom . .

4. $R$ decommits the commitment of the first step and reveal $\Gamma$.

5. For each $j \in \Gamma, C$ decommits the $\mathrm{Com}_{1}$ and $\mathrm{CECom}_{2}$ commitments whose committed values are $s_{j}$. 
The committed value of CCACom C $_{0}$ is determined by the committed values of $\mathrm{Com}_{1}$. Thus, the running time of $\mathcal{O}$ is at most $o\left(T_{2}(n)\right) \cdot \operatorname{poly}(n) \ll T_{2}(n)$.

To prove the CCA security of the scheme, we consider a series of hybrid experiments.

In the first hybrid, in the left interaction the committed value $\Gamma$ of CCACom $_{3}^{1: 1}$ is extracted by brute force and the committed value of CECom 2 is switched from $s_{j}$ to 0 for every $j \notin \Gamma$. Note that, during the CECom 2 commitments of the left, the combined running time of $\mathcal{A}$ and $\mathcal{O}$ is at most $T_{2}(n)$. Thus, from the hiding property of $\mathrm{CECom}_{2}$, the view of $\mathcal{A}$ in the first hybrid is indistinguishable from that of $\mathcal{A}$ in the honest experiment.

The second hybrid is the same as the first one except for the following: in every right session of which the second step ends after the start of the second step of the left session, the committed values of the CECom commitments are extracted; then, the answer of $\mathcal{O}$ are computed from the extracted values (instead of the committed values of $\mathrm{Com}_{1}$ ). We note that, since the second hybrid differs from the first one only in how the answers of $\mathcal{O}$ are computed, to show the indistinguishability it suffices to show that in the first hybrid the committed values of CECom 2 agree with those of $\mathrm{Com}_{1}$ in "most" indexes in every right session. We first note that if we ignore the messages that $\mathcal{A}$ receives in the left session, we can prove that the committed values of CECom 2 agree with those of $\mathrm{Com}_{1}$ in most indexes by using the property of the cut-and-choose technique. In the hybrid, however, $\mathcal{A}$ receives messages in the left session, in which $\Gamma$ is

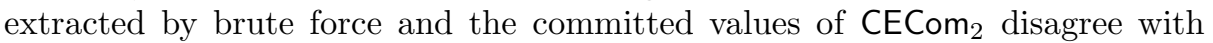
those of $\mathrm{Com}_{1}$ in $90 \%$ of indexes. Thus, $\mathcal{A}$ may be able to use the messages in the left to break the hiding property of $\mathrm{CCACom}^{1: 1}$ in the right. (Note that, if $\mathcal{A}$ can break the hiding property of CCACom ${ }^{1: 1}$, we cannot use the property of the cut-and-choose technique.) We show that $\mathcal{A}$ cannot break the hiding property of CCACom ${ }^{1: 1}$ even with the messages of the left session. A key is that given $\Gamma$, the left session can be simulated in polynomial time. Hence, one-one CCA security of CCACom ${ }^{1: 1}$ guarantees that the messages of the left session are useless for breaking the hiding property of CCACom ${ }^{1: 1}$. Thus, even with messages of the left session, the cut-and-choose guarantees that the committed values of CECom 2 agree with those of $\mathrm{Com}_{1}$ in most indexes. The view of $\mathcal{A}$ in the second hybrid is therefore indistinguishable from that of $\mathcal{A}$ in the first one.

The third hybrid is the same as the first one except that in the left session, the committed value of $\mathrm{Com}_{1}$ is switched from $s_{j}$ to 0 for every $j \notin \Gamma$. Note that during the Com $_{1}$ commitments of the left, the combined running time of $\mathcal{A}$ and $\mathcal{O}$ is at most $T_{0}(n) \cdot \operatorname{poly}\left(n^{\log n}\right) \ll T_{1}(n)$. This is because

- for every right session in which $\mathcal{A}$ completes the second step before the start of the second step of the left session, the answer of $\mathcal{O}$ (i.e., the committed value of $\mathrm{CCACom}_{0}$ ) can be computed before the start of $\mathrm{Com}_{1}$ commitments of the left session, and

- for every right session in which $\mathcal{A}$ completes the second step after the start of the second step of the left session, the answer of $\mathcal{O}$ is computed by 
extracting the committed values of $\mathrm{CECom}_{2}$, which requires rewinding $\mathcal{A}$ at most poly $\left(n^{\log n}\right)$ times.

Thus, from the hiding property of $\mathrm{Com}_{1}$, the view of $\mathcal{A}$ in the third hybrid is indistinguishable from that of $\mathcal{A}$ in the second one.

Note that, since $s$ is $(n+1)$-out-of- $10 n$ secret sharing, $\mathcal{A}$ receives no information of $v$ in the third hybrid. Thus, the view of $\mathcal{A}$ in the third hybrid is independent of $v$, and thus the CCA security follows.

\section{Preliminaries}

In this section, we explain the assumptions and the definitions that we use in this paper.

\subsection{Assumptions}

For our CCA-secure commitment scheme, we use a one-way function $f$ that is secure against $2^{n^{\epsilon}}$-time adversaries, where $\epsilon<1$ is a positive constant. Without loss of generality, we assume that $f$ can be inverted in time $2^{n}$. Let $T_{i}(n) \stackrel{\text { def }}{=}$ $2^{(\log n)^{(2 / \epsilon)^{10 i+1}}}$ for $i \in \mathbb{N}$. Then, by setting the security parameter of $f$ to $\ell_{i}(n)=$ $(\log n)^{(2 / \epsilon)^{10 i+2}}$, we obtain a one-way function $f_{i}$ that is secure against $T_{i}(n)$ time adversaries but can be inverted in time less than $T_{i+0.5}(n)$. We note that when $i \leq O(\log \log n)$, we have $\ell_{i}(n) \leq \operatorname{poly}(n)$.

For our composable MPC protocol, we additionally use semi-honest oblivious transfer protocols that are secure against $2^{\text {poly( } \log n)}$-time adversaries.

\subsection{Shamir's Secret Sharing Scheme}

In this paper, we use Shamir's $(n+1)$-out-of-10n secret sharing scheme. For any positive real number $x \leq 1$ and any $s=\left(s_{1}, \ldots, s_{10 n}\right)$ and $\boldsymbol{s}^{\prime}=\left(s_{1}^{\prime}, \ldots, s_{10 n}^{\prime}\right)$, we say that $s$ and $s^{\prime}$ are $x$-close if $\left|\left\{i \mid s_{i}=s_{i}^{\prime}\right\}\right| \geq x \cdot 10 n$. We note that Shamir's secret sharing is a codeword of Reed-Solomon code with minimum relative distance 0.9 . Thus, for any $x>0.55$ and any $s$ that is $x$-close to a valid codeword $\boldsymbol{w}$, we can compute $\boldsymbol{w}$ from $\boldsymbol{s}$.

\subsection{Commitment Schemes}

Recall that commitment schemes are two-party protocols between the committer $C$ and the receiver $R$. A transcript of the commit phase is accepted if $R$ does not abort in the commit phase. A transcript of the commit phase is valid if there exists a valid decommitment of this transcript. We use a 2-round statistically binding commitment scheme Com based on one-way functions [27. 
Strong computational binding property. We say that a commitment scheme $\langle C, R\rangle$ satisfies a strong computational binding property if for any PPT committer $\mathcal{C}^{*}$ interacting with the honest receiver $R$, the probability that $\mathcal{C}^{*}$ generates a commitment that has more than one committed value is negligible 5

\subsection{Extractable Commitments}

Roughly speaking, a commitment scheme is extractable if there exists an expected polynomial-time oracle machine (or extractor) $E$ such that for any committer $\mathcal{C}^{*}, E^{\mathcal{C}^{*}}$ extracts the value that $\mathcal{C}^{*}$ commits to whenever the commitment is valid. We note that when the commitment is invalid, $E$ may output a garbage value. (This is called over-extraction.)

There exists a 4-round extractable commitment scheme ExtCom based on one-way functions 33. The commit phase of ExtCom consists of three stagescommit, challenge, and reply - and given two accepted transcripts that have the same commit message but have different challenge messages, we can extract the committed value. Thus, we can extract the committed value by rewinding the committer and obtaining two such transcripts. In the following, we use slot to denote a pair of the challenge and reply messages in ExtCom. As shown in 33, ExtCom is in fact parallel extractable. Thus, even when a committer commits to many values in parallel, we can extract all committed values.

\subsection{Concurrently Extractable Commitments}

Roughly speaking, a commitment scheme is concurrently extractable if there exists an expected polynomial-time extractor $E$ such that for any committer $\mathcal{C}^{*}$ that concurrently commits to many values, $E^{\mathcal{C}^{*}}$ extracts the committed value of each commitment immediately after $\mathcal{C}^{*}$ generates each commitment.

Micciancio et al. 26] showed a concurrently extractable commitment CECom, which consists of $r$ executions of ExtCom, where $r$ is a parameter (see Figure 1). Note that CECom has $r$ sequential slots. Then, by using the rewinding strategy of [35, the committed values of CECom are concurrently extractable when $r=$ $\omega(\log n)$.

\section{Concurrently $T(n)$-Extractable Commitments}

For any function $T(n)$, we consider a relaxed notion of concurrent extractability called concurrent $T(n)$-extractability, which is the same as concurrent extractability except that the expected running time of the extractor is $T(n)$.

By using the rewinding strategy of 32 , we can show that CECom is concurrently poly $\left(n^{\log n}\right)$-extractable when $r \geq 3$. Note that in the stand-alone setting, we can extract the committed value of CECom by rewinding any single slot.

\footnotetext{
${ }^{5}$ The standard computational binding property guarantees only that for any PPT committer $\mathcal{C}^{*}$, the commitment that $\mathcal{C}^{*}$ generates cannot be decommitted to more than one value in polynomial time. Thus, this commitment may have more than one committed value.
} 
Commit Phase. The committer $C$ and the receiver $R$ receive common inputs $1^{n}$ and parameter $r$. To commit to $v \in\{0,1\}^{n}$, the committer $C$ does the following.

Step 1. $C$ and $R$ execute commit stage of ExtCom $r$ times in parallel.

Step $2 i(i \in[r]) . R$ sends the challenge message of ExtCom for the $i$-th session.

Step $2 i+1(i \in[r]) . C$ sends the reply message of ExtCom for the $i$-th session.

Decommit Phase. $C$ sends $v$ to $R$ and decommits all the ExtCom commitments in the commit phase.

Fig. 1. Concurrently extractable commitment CECom 26$]$

\subsection{Trapdoor Commitments}

Roughly speaking, trapdoor commitments are ones such that there exists a simulator that can generate a simulated commitment and can later decommit it to any value.

Pass and Wee 33] showed that the black-box protocol TrapCom in Figure 2 is a trapdoor bit commitment scheme. In fact, given the receiver's challenge $e$ in advance, we can generate a simulated commitment and decommit it to both 0 and 1 in a straight-line manner (i.e., without rewinding the receiver) as follows. To generate a simulated commitment, the simulator internally simulates an interaction between $C$ and $\mathcal{R}^{*}$ honestly except that in Step 2, the simulator chooses random $\gamma \in\{0,1\}$ and lets each $v_{i}$ be a matrix such that the $e_{i}$-th row of $v_{i}$ is $\left(\eta_{i}, \eta_{i}\right)$ and the $\left(1-e_{i}\right)$-th row of $v_{i}$ is $\left(\gamma \oplus \eta_{i},(1-\gamma) \oplus \eta_{i}\right)$. To decommit the simulated commitment to $\sigma \in\{0,1\}$, the simulator decommits all the commitments in the $(\sigma \oplus \gamma)$-th column of each $v_{i}$.

From the extractability of ExtCom, we can show that TrapCom is extractable. In addition, by using the hiding property of Com, we can show that TrapCom satisfies the strong computational binding property. (Roughly speaking, if $\mathcal{C}^{*}$ generates a commitment that has more than one committed value, we can compute the committed value $e$ of Com by extracting $v_{1}, \ldots, v_{n}$.)

Pass and Wee 33 showed that by running TrapCom in parallel, we obtain a black-box trapdoor commitment PTrapCom for multiple bits. PTrapCom also satisfies the strong computational binding property and extractability.

\subsection{CCA-Secure Commitments}

We recall the definition of CCA security and $\kappa$-robustness [7, 23]. Tag-based commitment schemes are ones such that both the committer and the receiver receive a string, or tag, as an additional input. 
Commit Phase. To commit to $\sigma \in\{0,1\}$ on common input $1^{n}$, the committer $C$ does the following with the receiver $R$ :

Step 1. $R$ chooses a random $n$-bit string $e=\left(e_{1}, \ldots, e_{n}\right)$ and commits to $e$ by using Com.

Step 2. For each $i \in[n]$, the committer $C$ chooses a random $\eta_{i} \in\{0,1\}$ and sets

$$
v_{i}:=\left(\begin{array}{cc}
v_{i}^{00} & v_{i}^{01} \\
v_{i}^{10} & v_{i}^{11}
\end{array}\right)=\left(\begin{array}{cc}
\eta_{i} & \eta_{i} \\
\sigma \oplus \eta_{i} & \sigma \oplus \eta_{i}
\end{array}\right) .
$$

Then, for each $i \in[n], \alpha \in\{0,1\}$, and $\beta \in\{0,1\}$ in parallel, $C$ commits to $v_{i}^{\alpha \beta}$ by using ExtCom; let $\left(v_{i}^{\alpha \beta}, d_{i}^{\alpha \beta}\right)$ be the corresponding decommitment.

Step 3. $R$ decommits the Step 1 commitment to $e$.

Step 4. For each $i \in[n], C$ sends $\left(v_{i}^{e_{i} 0}, d_{i}^{e_{i} 0}\right)$ and $\left(v_{i}^{e_{i} 1}, d_{i}^{e_{i} 1}\right)$ to $R$. Then, $R$ checks whether these are valid decommitments and whether $v_{i}^{e_{i} 0}=v_{i}^{e_{i} 1}$.

Decommit Phase. $C$ sends $\sigma$ and random $\gamma \in\{0,1\}$ to $R$. In addition, for every $i \in[n], C$ sends $\left(v_{i}^{0 \gamma}, d_{i}^{0 \gamma}\right)$ and $\left(v_{i}^{1 \gamma}, d_{i}^{1 \gamma}\right)$ to $R$. Then, $R$ checks whether $\left(v_{i}^{0 \gamma}, d_{i}^{0 \gamma}\right)$ and $\left(v_{i}^{1 \gamma}, d_{i}^{1 \gamma}\right)$ are valid decommitments for every $i \in[n]$ and whether $v_{0}^{0 \gamma} \oplus v_{0}^{1 \gamma}=\cdots=v_{n}^{0 \gamma} \oplus v_{n}^{1 \gamma}=\sigma$.

Fig. 2. Black-box trapdoor bit commitment TrapCom

CCA Security (w.r.t. the Committed-value Oracle). Roughly speaking, a tag-based commitment scheme $\langle C, R\rangle$ is $C C A$-secure if the hiding property of $\langle C, R\rangle$ holds even against adversary $\mathcal{A}$ that interacts with the committed-value oracle during the interaction with the committer. The committed-value oracle $\mathcal{O}$ interacts with $\mathcal{A}$ as an honest receiver in many concurrent sessions of the commit phase of $\langle C, R\rangle$ using tags chosen adaptively by $\mathcal{A}$. At the end of each session, if the commitment of this session is invalid or has multiple committed values, $\mathcal{O}$ returns $\perp$ to $\mathcal{A}$. Otherwise, $\mathcal{O}$ returns the unique committed value to $\mathcal{A}$.

More precisely, let us consider the following probabilistic experiment $\operatorname{IND}_{b}(\langle C, R\rangle, \mathcal{A}, n, z)$ for each $b \in\{0,1\}$. On input $1^{n}$ and auxiliary input $z$, adversary $\mathcal{A}^{\mathcal{O}}$ adaptively chooses a pair of challenge values $v_{0}, v_{1} \in\{0,1\}^{n}$ and an $n$-bit tag id $\in\{0,1\}^{n}$. Then, $\mathcal{A}^{\mathcal{O}}$ receives a commitment to $v_{b}$ with tag id, and $\mathcal{A}$ outputs $y$. The output of the experiment is $\perp$ if during the experiment, $\mathcal{A}$ sends $\mathcal{O}$ any commitment using tag id. Otherwise, the output of the experiment is $y$. Let $\operatorname{IND}_{b}(\langle C, R\rangle, \mathcal{A}, n, z)$ denote the output of experiment $\operatorname{IND}_{b}(\langle C, R\rangle, \mathcal{A}, n, z)$.

Then, the CCA security of $\langle C, R\rangle$ is defined as follows.

Definition 1. Let $\langle C, R\rangle$ be a tag-based commitment scheme and $\mathcal{O}$ be the committed-value oracle of $\langle C, R\rangle$. Then, $\langle C, R\rangle$ is CCA-secure (w.r.t the 
committed-value oracle) if for any $\mathrm{PPT}$ adversary $\mathcal{A}$, the following are computationally indistinguishable:

$-\left\{\operatorname{IND}_{0}(\langle C, R\rangle, \mathcal{A}, n, z)\right\}_{n \in \mathbb{N}, z \in\{0,1\}^{*}}$

- $\left\{\operatorname{IND}_{1}(\langle C, R\rangle, \mathcal{A}, n, z)\right\}_{n \in \mathbb{N}, z \in\{0,1\}^{*}}$

If the length of the tags chosen by $\mathcal{A}$ is $t(n)$ instead of $n,\langle C, R\rangle$ is CCA-secure for tags of length $t(n)$.

We also consider a relaxed notion of CCA security called one-one CCA security. In the definition of one-one CCA security, we consider adversaries that interact with $\mathcal{O}$ only in a single session of the commit phase.

In the following, we use left session to denote the session of the commit phase between the committer and $\mathcal{A}$, and use right sessions to denote the sessions between $\mathcal{A}$ and $\mathcal{O}$.

$\kappa$-robustness (w.r.t. the Committed-value Oracle). Roughly speaking, a tag-based commitment scheme is $\kappa$-robust if for any adversary $\mathcal{A}$ and any ITM $B$, the joint output of a $\kappa$-round interaction between $\mathcal{A}^{\mathcal{O}}$ and $B$ can be simulated without $\mathcal{O}$ by a PPT simulator. Thus, the $\kappa$-robustness guarantees that the committed-value oracle is useless in attacking any $\kappa$-round protocol.

Formally, let $\langle C, R\rangle$ be a tag-based commitment scheme and $\mathcal{O}$ be the committed-value oracle of $\langle C, R\rangle$. For any constant $\kappa \in \mathbb{N}$, we say that $\langle C, R\rangle$ is $\kappa$-robust (w.r.t. the committed value oracle) if there exists a PPT oracle machine (or simulator) $\mathcal{S}$ such that for any PPT adversary $\mathcal{A}$ and any $\kappa$-round PPT ITM $B$, the following are computationally indistinguishable:

- output $\left._{B, \mathcal{A}^{\mathcal{O}}}\left[\left\langle B(y), \mathcal{A}^{\mathcal{O}}(z)\right\rangle\left(1^{n}, x\right)\right]\right\}_{n \in \mathbb{N}, x, y, z \in\{0,1\}^{n}}$

- output $\left._{B, \mathcal{S} \mathcal{A}}\left[\left\langle B(y), \mathcal{S}^{\mathcal{A}}(z)\right\rangle\left(1^{n}, x\right)\right]\right\}_{n \in \mathbb{N}, x, y, z \in\{0,1\}^{n}}$

Here, for any ITM $A$ and $B$, we use output ${ }_{A, B}[\langle A(y), B(z)\rangle(x)]$ to denote the joint output of $A$ and $B$ in an interaction between them on inputs $x, y$ to $A$ and $x, z$ to $B$ respectively.

We also consider a relaxed notion of $\kappa$-robustness called $\kappa$-PQT-robustness. In the definition of $\kappa$-PQT-robustness, we allow the simulator to run in quasipolynomial time.

\section{One-One CCA Security for Short Tags}

In this section, we construct a one-one CCA-secure commitment for tags of length $O(\log \log \log n)$. (Due to lack of space, the full proof is deferred to the full version.) Since the length of the tags is $O(\log \log \log n)$, we can view each tag as a value in $\{0,1 \ldots, d-1=O(\log \log n)\}$. 


\section{$4.1 \quad$ Building Blocks}

Let $T_{i}(n) \stackrel{\text { def }}{=} 2^{(\log n)^{(2 / \epsilon)^{10 i+1}}}$ for $i \in \mathbb{N}$. Then, for constants $a, b \in \mathbb{N}$, PTrapCom $_{a}^{b}$ is a commitment scheme such that

- the hiding property holds against any $T_{a}(n)$-time adversary but is completely broken in time $T_{a+0.5}(n)$,

- the strong computational binding property holds against any $T_{b}(n)$-time adversary, and

- there exists a $T_{b+0.5}(n)$-time straight-line simulator (of the trapdoor property) such that the simulated commitment is indistinguishable from the actual commitment in time $T_{a}(n)$. (This holds even when $T_{b+0.5}(n) \gg T_{a}(n)$.)

We can construct PTrapCom ${ }_{a}^{b}$ by appropriately setting the security parameters of Com and ExtCom in PTrapCom.

PCETrapCom ${ }_{a}^{b}$ is the same as PTrapCom ${ }_{a}^{b}$ except that we use CECom in Step 2 instead of ExtCom.

\subsection{One-One CCA Security for Tags of Length $O(\log \log \log n)$}

Lemma 1. Let $\epsilon<1$ be a positive constant, and for any $i \in \mathbb{N}$, let $T_{i}(n) \stackrel{\text { def }}{=}$ $2^{(\log n)^{(2 / \epsilon)^{10 i+1}}}$. Assume the existence of one-way functions that are secure against $2^{n^{\epsilon}}$-time adversaries. Then, for any $i \in \mathbb{N}$, there exists a constant-round commitment scheme CCACom ${ }_{i}^{1: 1}$ that satisfies the following for any $T_{i}(n)$-time adversary.

- Strong computational binding property, and

- One-one CCA security for tags of length $O(\log \log \log n)$.

Furthermore, $\mathrm{CCACom}_{i}^{1: 1}$ uses the underlying one-way function only in a blackbox way.

Proof. CCACom ${ }_{i}^{1: 1}$ is shown in Figure 3. The binding property follows from that of PTrapCom $i+d+1$. Thus, it remains to show that CCACom ${ }_{i}^{1: 1}$ is one-one CCA secure for tags of length $O(\log \log \log n)$.

To show that CCACom $1: 1$ is one-one CCA secure, we show that for any $T_{i}(n)$ time adversary $\mathcal{A}$ that interacts with $\mathcal{O}$ only in a single session, the following are computationally indistinguishable:

$$
\begin{aligned}
& -\left\{\operatorname{IND}_{0}\left(\mathrm{CCACom}_{i}^{1: 1}, \mathcal{A}, n, z\right)\right\}_{n \in \mathbb{N}, z \in\{0,1\}^{*}} \\
& -\left\{\operatorname{IND}_{1}\left(\mathrm{CCACom}_{i}^{1: 1}, \mathcal{A}, n, z\right)\right\}_{n \in \mathbb{N}, z \in\{0,1\}^{*}}
\end{aligned}
$$

At the end of the right session, the committed-value oracle $\mathcal{O}$ does the following. First, $\mathcal{O}$ computes the committed values $s=\left(s_{1}, \ldots, s_{10 n}\right)$ of the Stage 1 commitments by brute force. (If the committed value of the $j$-th commitment is not uniquely determined, $s_{j}$ is defined to be $\perp$.) Then, $\mathcal{O}$ checks whether the following conditions hold: (1) $\boldsymbol{s}$ is 0.9 -close to a valid codeword $\boldsymbol{w}=\left(w_{1}, \ldots, w_{10 n}\right)$ and (2) for every $j \in \Gamma$ (where $\Gamma$ is the subset that $\mathcal{O}$ sends to $\mathcal{A}$ in Stage 4 ), 
Commit Phase. The committer $C$ and the receiver $R$ receive common inputs $1^{n}$ and id $\in\{0,1, \ldots, d-1=O(\log \log n)\}$. To commit to $v \in\{0,1\}^{n}$, the committer $C$ does the following with the receiver $R$.

Stage 1. $C$ computes an $(n+1)$-out-of-10n Shamir's secret sharing $s=$ $\left(s_{1}, \ldots, s_{10 n}\right)$ of value $v$. Then, for each $j \in[10 n]$ in parallel, $C$ commits to $s_{j}$ by using PTrapCom $i+d+1$. Let $\left(s_{j}, d_{j}\right)$ be the decommitment of the $j$-th commitment.

Stage 2. For each $j \in[10 n]$ in parallel, $C$ commits to $\left(s_{j}, d_{j}\right)$ by using PCETrapCom $i+d+2$. Here, the number of slots in PCETrapCom $\operatorname{lid}_{i+d+2}^{i+\mathrm{id}+1}$ is $\max (3, r+1)$, where $r$ is the round complexity of PTrapCom $i+d+1$ in Stage 1.

Stage 3. For each $j \in[10 n]$ in parallel, $C$ commits to $\left(s_{j}, d_{j}\right)$ by using PCETrapCom $i+d-i d$. Here, the number of slots in PCETrapCom PCo $_{i+d+2}^{i+d-i d}$ is $\max (3, r+1)$.

Stage 4. $R$ sends a random subset $\Gamma \subseteq[10 n]$ of size $n$ to $C$.

Stage 5. For each $j \in \Gamma, C$ decommits the $j$-th Stage 2 commitment and the $j$-th Stage 3 commitment to $\left(s_{j}, d_{j}\right)$. Then, $R$ checks whether $\left(s_{j}, d_{j}\right)$ is a valid decommitment of the $j$-th Stage 1 commitment.

Decommit Phase. $C$ sends $v, s=\left(s_{1}, \ldots, s_{10 n}\right)$, and $\boldsymbol{d}=\left(d_{1}, \ldots, d_{10 n}\right)$ to $R$. Then, $R$ checks whether $\left(s_{j}, d_{j}\right)$ is a valid decommitment of the $j$-th Stage 1 commitment for every $j \in[10 n]$. Furthermore, $R$ checks whether (1) $\boldsymbol{s}$ is 0.9 -close to a valid codeword $\boldsymbol{w}=\left(w_{1}, \ldots, w_{10 n}\right)$ and (2) for each $j \in \Gamma, w_{j}$ is equal to the share that was revealed in Stage 5. Finally, $R$ checks whether $\boldsymbol{w}$ is a codeword corresponding to $v$.

Fig. 3. One-one CCA-secure commitment $\operatorname{CCACom}_{i}^{1: 1}$

$w_{j}$ is equal to the share that was revealed in Stage 5 . If both conditions hold, $\mathcal{O}$ recovers $v$ from $\boldsymbol{w}$ and returns $v$ to $\mathcal{A}$. Otherwise, $\mathcal{O}$ returns $v:=\perp$ to $\mathcal{A}$. We note that the running time of $\mathcal{O}$ is at most poly $(n) \cdot T_{i+d+1.5}(n)$.

To show the indistinguishability, we consider hybrid experiments $G_{a}^{b}(n, z)$ for $a \in\{0,1,2,3\}$ and $b \in\{0,1\}$.

Hybrid $G_{0}^{b}(n, z)$ is the same as experiment $\operatorname{IND}_{b}\left(\operatorname{CCACom}_{i}^{1: 1}, \mathcal{A}, n, z\right)$.

Hybrid $G_{1}^{b}(n, z)$ is the same as $G_{0}^{b}(n, z)$ except for the following:

- In Stage 2 (resp., Stage 3) on the left, the left committer simulates the $10 n$ commitments of PCETrapCom $i+d+2$ (resp., PCETrapCom $i+d+2$ ) by using the straight-line simulator.

- In Stage 5 on the left, for each $j \in \Gamma$, the left committer decommits the simulated commitment of PCETrapCom $i+d+2$ (resp., PCETrapCom $i+d+2$-id to $\left(s_{j}, d_{j}\right)$ by using the simulator.

We note that the running time of $G_{1}^{b}(n, z)$ is at most poly $(n) \cdot T_{i+d+1.5}(n)$. Hybrid $G_{2}^{b}(n, z)$ is the same as $G_{1}^{b}(n, z)$ except for the following: 
- Let $\widetilde{i d}$ be the tag of the right session. In Stage 2 (resp., Stage 3) of the right session, the committed values of the PCETrapCom $i+d+2$ (resp., PCETrapCom $\left.{ }_{i+d+2}^{i+d-\tilde{\mathrm{id}}}\right)$ commitments are extracted without rewinding Stage 1 on the left by using the technique of [7,22. (That is, in Step 2 of each PCETrapCom ${ }_{i+d+2}^{i+\tilde{\mathrm{id}}+1}$ (resp. PCETrapCom ${ }_{i+d+2}^{i+d-\tilde{\mathrm{id}}}$ ) commitment, the committed values of CECom are extracted by rewinding a single slot that does not contain any Stage 1 messages of the left session. Such a slot must exist, since the number of slots in CECom is $\max (3, r+1)$.) Then, $\widehat{\boldsymbol{s}}=\left(\widehat{s}_{1}, \ldots, \widehat{s}_{10 n}\right)$ is defined as follows: if there exists $a \in\{2,3\}$ such that the extracted value $\left(\widehat{s}_{j}^{(a)}, \widehat{d}_{j}^{(a)}\right)$ of the $j$-th commitment in Stage $a$ is a valid decommitment of the $j$-th commitment in Stage 1 , let $\widehat{s}_{j} \stackrel{\text { def }}{=} \widehat{s}_{j}^{(a)}$ (if $\operatorname{both}\left(\widehat{s}_{j}^{(2)}, \widehat{d}_{j}^{(2)}\right)$ and $\left(\widehat{s}_{j}^{(3)}, \widehat{d}_{j}^{(3)}\right)$ are valid decommitments but $\widehat{s}_{j}^{(2)} \neq \widehat{s}_{j}^{(3)}$, let $\widehat{s}_{j} \stackrel{\text { def }}{=} \perp$ ); otherwise, let $\widehat{s}_{j} \stackrel{\text { def }}{=} \perp$.

- At the end of the right session, $\mathcal{O}$ checks whether the following conditions hold: (1) $\widehat{\boldsymbol{s}}$ is 0.8 -close to a valid codeword $\widehat{\boldsymbol{w}}=\left(\widehat{w}_{1}, \ldots, \widehat{w}_{10 n}\right)$ and $(2)$ for every $j \in \Gamma, \widehat{w}_{j}$ is equal to the share that was revealed in Stage 5. If both conditions hold, $\mathcal{O}$ recovers $\widehat{v}$ from $\widehat{\boldsymbol{w}}$ and returns $\widehat{v}$ to $\mathcal{A}$. Otherwise, $\mathcal{O}$ returns $\widehat{v}:=\perp$ to $\mathcal{A}$. We note that $\mathcal{O}$ does not extract the committed values of the Stage 1 commitments.

We note that the expected running time of $G_{2}^{b}(n, z)$ is poly $(n) \cdot T_{i+d+0.5}(n)$. Hybrid $G_{3}^{b}(n, z)$ is the same as $G_{2}^{b}(n, z)$ except that on the left, the Stage 1 commitments are simulated by the straight-line simulator of PTrapCom $i+d+1$.

Since $\mathcal{A}$ receives no information about $\left\{s_{j}\right\}_{j \notin \Gamma}$ in $G_{3}^{0}(n, z)$ and $G_{3}^{1}(n, z)$, the output of $G_{3}^{0}(n, z)$ and that of $G_{3}^{1}(n, z)$ are identically distributed. Then, we consider the following claims. In what follows, we use $\mathrm{G}_{i}^{b}(n, z)$ to denote the output of experiment $G_{i}^{b}(n, z)$.

Claim 1. For each $b \in\{0,1\},\left\{\mathrm{G}_{0}^{b}(n, z)\right\}_{n \in \mathbb{N}, z \in\{0,1\}^{*}}$ and $\left\{\mathrm{G}_{1}^{b}(n, z)\right\}_{n \in \mathbb{N}, z \in\{0,1\}^{*}}$ are computationally indistinguishable.

Claim 2. For each $b \in\{0,1\},\left\{\mathrm{G}_{1}^{b}(n, z)\right\}_{n \in \mathbb{N}, z \in\{0,1\}^{*}}$ and $\left\{\mathrm{G}_{2}^{b}(n, z)\right\}_{n \in \mathbb{N}, z \in\{0,1\}^{*}}$ are statistically indistinguishable.

Claim 3. For each $b \in\{0,1\},\left\{\mathrm{G}_{2}^{b}(n, z)\right\}_{n \in \mathbb{N}, z \in\{0,1\}^{*}}$ and $\left\{\mathrm{G}_{3}^{b}(n, z)\right\}_{n \in \mathbb{N}, z \in\{0,1\}^{*}}$ are computationally indistinguishable.

The lemma follows from these claims.

Proof (of Claim 11). $G_{1}^{b}(n, z)$ differs from $G_{0}^{b}(n, z)$ only in that the Stage 2 commitments and the Stage 3 commitments on the left are simulated by the simulator of PCETrapCom $i+d+2$ and that of PCETrapCom $i+d+2$. Then, since the running time of $G_{0}^{b}(n, z)$ and that of $G_{1}^{b}(n, z)$ are at most poly $(n) \cdot T_{i+d+1.5}(n) \ll$ $T_{i+d+2}(n)$, the claim follows from the trapdoor property of PCETrapCom $i+d+2$ and that of PCETrapCom $i+d+2$. 
Next, we consider Claim 2 Note that $G_{2}^{b}(n, z)$ differs from $G_{1}^{b}(n, z)$ in that $\mathcal{O}$ computes the committed value of the right session from the extracted values of the Stage 2 commitments and those of the Stage 3 commitments instead of from those of Stage 1 commitments. We prove Claim 2 by showing that in the right session of $G_{2}^{b}(n, z)$, the value $\widehat{v}$ that $\mathcal{O}$ computes is the same as the value $v$ that $\mathcal{O}$ computes in $G_{1}^{b}(n, z)$. Toward this end, we first show that in the right session of $G_{1}^{b}(n, z)$, the strong computational binding property holds in Stage 1 and either in Stage 2 or in Stage 3. (Note that from the property of the cutand-choose technique, this implies that the committed values of either the Stage 2 commitments or the Sage 3 commitments are 0.9-close to those of the Stage 1 commitments except with negligible probability.) Let us say that $\mathcal{A}$ cheats in Stage 1 if at least one of $10 n$ PTrapCom commitments in Stage 1 on the right has more than one committed value. We define cheating in Stage 2 and cheating in Stage 3 similarly. Then, we prove two subclaims.

Subclaim 1. In $G_{1}^{b}(n, z)$, the probability that $\mathcal{A}$ cheats in Stage 1 is negligible.

Proof (sketch). This subclaim follows directly from the strong computational binding property of $\mathrm{PTrapCom} i+d+1$, since the running time of $G_{1}^{b}(n, z)$ is at most poly $(n) \cdot T_{i+d+0.5}(n) \ll T_{i+d+1}(n)$ when $\mathcal{A}$ completes Stage 1 on the right.

Subclaim 2. In $G_{1}^{b}(n, z)$, the probability that $\mathcal{A}$ cheats in Stage 2 and Stage 3 simultaneously is negligible.

Proof (sketch). To prove this subclaim, we need to show that even though the left committer "cheats," $\mathcal{A}$ cannot use the messages received on the left to cheat on the right. This can be proven by following the proof of the scheme of [34]. Roughly speaking, we show that there always exists $a^{*} \in\{2,3\}$ such that during Stage $a^{*}$ on the right, the left session can be simulated in "short" time (i.e., the left session can be simulated without breaking the strong computational binding property of PCETrapCom in Stage $a^{*}$ ). A little more precisely, we show the following. Recall that the commitment of PCETrapCom can be simulated in polynomial time if we know the committed value of the Step 1 commitment of PCETrapCom. Then, we show that in the left session, either this committed value can be extracted in "short" time (during Stage $a^{*}$ of the right session) or it can be extracted before $\mathcal{A}$ starts Stage $a^{*}$ on the right (and thus can be considered as an auxiliary input). Once we show that $\mathcal{A}$ cannot use the messages received on the left to cheat on the right, the subclaim follows from the strong computational binding property of PCETrapCom on the right.

Now, we are ready to prove Claim 2 ,

Proof (sketch of Claim Q). As noted above, we prove Claim 2 by showing that in the right session, the value computed by $\mathcal{O}$ in $G_{2}^{b}(n, z)$ is equal to the value computed by $\mathcal{O}$ in $G_{1}^{b}(n, z)$. From Subclaim 2, there exists $a \in\{2,3\}$ such that the committed values of the Stage $a$ commitments are uniquely determined. Then, since the committed values of the Stage 1 commitments and those of 
Stage $a$ commitments are uniquely determined before $\Gamma$ is chosen, the committed values of the Stage 1 commitments and those of Stage $a$ commitments are 0.9close except with negligible probability. Then, since we have carefully defined the behavior of $\mathcal{O}$ in $G_{2}^{b}(n, z)$ (in particular, since $\mathcal{O}$ checks whether the share is 0.8 -close to a valid codeword in $\left.G_{2}^{b}(n, z)\right)$, we can show that the value computed by $\mathcal{O}$ from the extracted values of Stage 2 and 3 is the same as the one computed from the committed values of Stage 1 in a similar manner to the previous works on black-box constructions [9, 10, 23, 37.

Finally, we prove Claim 3 .

Proof (of Claim [3). $G_{3}^{b}(n, z)$ differs from $G_{2}^{b}(n, z)$ only in that on the left, the Stage 1 commitments and their decommitments are generated by the simulator of PTrapCom $i+d+1$. Then, since the running time of $G_{2}^{b}(n, z)$ and that of $G_{3}^{b}(n, z)$ are at most poly $(n) \cdot T_{i+d+0.5}(n) \ll T_{i+d+1}(n)$ except for Stage 1 on the left, and since Stage 1 on the left is not rewound in $G_{2}^{b}(n, z)$ and in $G_{3}^{b}(n, z)$, the claim follows from the trapdoor property of PTrapCom $i+d+1$.

\section{CCA Security from One-One CCA Security}

In this section, we show a transformation from any one-one CCA-secure commitment scheme to a CCA-secure commitment scheme. To use this transformation to obtain a general MPC protocol, we also show that the resultant CCA-secure commitment satisfies $\kappa$-PQT-robustness for any $\kappa \in \mathbb{N}$. (Due to lack of space, the full proof is deferred to the full version.)

Lemma 2. Let $\epsilon<1$ be a positive constant, and assume the existence of oneway functions that are secure against $2^{n^{\epsilon}}$-time adversaries. Let $r(\cdot)$ and $t(\cdot)$ be arbitrary functions, let $T_{i}(n) \stackrel{\text { def }}{=} 2^{(\log n)^{(2 / \epsilon)^{10 i+1}}}$ for any $i \in \mathbb{N}$, and let $\operatorname{CCACom}_{i+3}^{1: 1}$ be an $r(n)$-round commitment scheme that satisfies the following for any $T_{i+3}(n)$ time adversary.

- Strong computational binding property, and

- One-one CCA security for tags of length $t(n)$.

Then, for any $\kappa \in \mathbb{N}$, there exists an $(r(n)+O(1))$-round commitment scheme $\mathrm{CCACom}_{i}$ that satisfies the following for any $T_{i}(n)$-time adversary.

- Statistical binding property,

- CCA security for tags of length $t(n)$, and

$-\kappa$-PQT-robustness.

If $\mathrm{CCACom}_{i+3}^{1: 1}$ uses the underlying one-way function only in a black-box way, then $\mathrm{CCACom}_{i}$ uses the underlying one-way function only in a black-box way.

In the proof of Lemma 2, we use the following building blocks, which we can obtain by appropriately setting the security parameters of known protocols 26, 27, 32 . 
Commit Phase. The committer $C$ and the receiver $R$ receive common inputs $1^{n}$ and id $\in\{0,1\}^{t(n)}$. To commit to $v \in\{0,1\}^{n}$, the committer $C$ does the following with the receiver $R$.

Stage 1. $R$ chooses a random subset $\Gamma \subseteq[10 n]$ of size $n$. Then, $R$ commits to $\Gamma$ by using CCACom CCO3 $_{i+3}^{1}$ with tag id.

Stage 2. $C$ computes an $(n+1)$-out-of-10n Shamir's secret sharing $s=$ $\left(s_{1}, \ldots, s_{10 n}\right)$ of value $v$. Then, for each $j \in[10 n]$ in parallel, $C$ commits to $s_{j}$ by using $\operatorname{Com}_{i+1}$.

Stage 3. For each $j \in[10 n]$ in parallel, $C$ commits to $s_{j}$ by using CECom Com $_{i+2}$. Stage 4. $R$ decommits the Stage 1 commitment to $\Gamma$.

Stage 5. For every $j \in[10 n]$, let the $j$-th column denote the $j$-th commitment in Stage 2 and the $j$-th one in Stage 3 (that is, the commitments whose committed value is $s_{j}$ ). Then, for each $j \in \Gamma, C$ decommits the commitments of the $j$-th column to $s_{j}$.

Decommit Phase. $C$ sends $v$ to $R$ and decommits the Stage 2 commitments to $s$. Then, $R$ checks whether all of these decommitments are valid. Furthermore, $R$ checks whether (1) $s$ is 0.9 -close to a valid codeword $\boldsymbol{w}=\left(w_{1}, \ldots, w_{10 n}\right)$ and $(2)$ for every $j \in \Gamma, w_{j}$ is equal to the share that was revealed in Stage 5. Finally, $R$ checks whether $\boldsymbol{w}$ is a codeword corresponding to $v$.

Fig. 4. CCA-secure commitment CCACom $_{i}$

- A 2-round statistically binding commitment $\operatorname{Com}_{i+1}$ that is secure against $T_{i+1}(n)$-time adversaries but is completely broken in time $T_{i+1.5}(n)$.

- A constant-round concurrently poly $\left(n^{\log n}\right)$-extractable commitment CECom $_{i+2}$ that is secure against $T_{i+2}(n)$-time adversaries but is completely broken in time $T_{i+2.5}(n)$. The number of slots in CECom $_{i+2}$ is $\kappa+3$.

We note that both $\operatorname{Com}_{i+1}$ and $\mathrm{CECom}_{i+2}$ use the underlying one-way function in a black-box way.

Proof (of Lemma 2). CCACom ${ }_{i}$ is shown in Figure 4. The statistical binding property of $\mathrm{CCACom}_{i}$ follows from that of $\mathrm{Com}_{i+1}$. Then, we consider the following propositions.

Proposition 1. For any $T_{i}(n)$-time adversary, $\mathrm{CCACom}_{i}$ is $C C A$ secure for tags of length $t(n)$.

Proposition 2. For any $T_{i}(n)$-time adversary, CCACom CA $_{i}$-PQT-robust.

The lemma follows from these propositions.

Below, we prove Proposition 1] The proof of Proposition 2 is given in the full version. (Proposition 2 can be proven by extending the proof of Proposition [1) 
Proof (of Proposition 11). We show that for any $T_{i}(n)$-time adversary $\mathcal{A}$, the following are computationally indistinguishable:

- $\left\{\mathrm{IND}_{0}\left(\mathrm{CCACom}_{i}, \mathcal{A}, n, z\right)\right\}_{n \in \mathbb{N}, z \in\{0,1\}^{*}}$

- $\left\{\mathrm{IND}_{1}\left(\mathrm{CCACom}_{i}, \mathcal{A}, n, z\right)\right\}_{n \in \mathbb{N}, z \in\{0,1\}^{*}}$

Note that $\mathcal{O}$ does the following in each right session. First, $\mathcal{O}$ extracts the committed values $\boldsymbol{s}=\left(s_{1}, \ldots, s_{10 n}\right)$ of the Stage 2 commitments by brute force. (If the committed value of the $j$-th commitment is not uniquely determined, $s_{j}$ is defined to be $\perp$.) Then, at the end of the session, $\mathcal{O}$ checks whether the following conditions hold: (1) $\boldsymbol{s}$ is 0.9 -close to a valid codeword $\boldsymbol{w}=\left(w_{1}, \ldots, w_{10 n}\right)$, and (2) for every $j \in \Gamma$ (where $\Gamma$ is the value that $\mathcal{O}$ sends to $\mathcal{A}$ in Stage 4 ), $w_{j}$ is equal to the share that was revealed in Stage 5 . If both conditions hold, $\mathcal{O}$ recovers $v$ from $\boldsymbol{w}$ and returns $v$ to $A$. Otherwise, $\mathcal{O}$ returns $v:=\perp$ to $\mathcal{A}$. We note that the running time of $\mathcal{O}$ is at most poly $(n) \cdot T_{i+1.5}(n)$.

To show the indistinguishability, we consider hybrid experiments $H_{a}^{b}(n, z)$ for $a \in\{0,1,2,3\}$ and $b \in\{0,1\}$.

Hybrid $H_{0}^{b}(n, z)$ is the same as experiment $\operatorname{IND}_{b}\left(\operatorname{CCACom}_{i}, \mathcal{A}, n, z\right)$.

Hybrid $H_{1}^{b}(n, z)$ is the same as $H_{0}^{b}(n, z)$ except for the following:

- In Stage 1 of the left session, the committed value $\Gamma$ is extracted by brute force. If the commitment is invalid or has multiple committed values, $\Gamma$ is defined to be a random subset 6

- In Stage 3 of the left session, the left committer commits to 0 instead of $s_{j}$ for each $j \notin \Gamma$.

The running time of $H_{1}^{b}(n, z)$ is at most poly $(n) \cdot T_{i+1.5}(n)$ except for the brute-force extraction of the Stage 1 commitment on the left.

Hybrid $H_{2}^{b}(n, z)$ is the same as $H_{1}^{b}(n, z)$ except for the following:

- In every right session of which Stage 2 ends after $\mathcal{A}$ starts Stage 2 on the left, the committed values of the Stage 3 commitments are extracted by using the concurrent poly $\left(n^{\log n}\right)$-extractability of CECom $\operatorname{CE}_{i+2}$. Let $\widehat{\boldsymbol{s}}=$ $\left(\widehat{s}_{1}, \ldots, \widehat{s}_{10 n}\right)$ be the extracted values, where $\widehat{s}_{j}$ is defined to be $\perp$ if the extraction of the $j$-th commitment fails.

- At the end of each right session in which $\widehat{\boldsymbol{s}}=\left(\widehat{s}_{1}, \ldots, \widehat{s}_{10 n}\right)$ is extracted, $\mathcal{O}$ does the following. First, $\mathcal{O}$ checks whether the following conditions hold: (1) $\widehat{\boldsymbol{s}}$ is 0.8 -close to a valid codeword $\widehat{\boldsymbol{w}}=\left(\widehat{w}_{1}, \ldots, \widehat{w}_{10 n}\right)$ and $(2)$ for every $j \in \widetilde{\Gamma}$ (where $\widetilde{\Gamma}$ is the value that $\mathcal{O}$ sends to $\mathcal{A}$ in this session), $\widehat{w}_{j}$ is equal to the share that was revealed in Stage 5 . If both conditions hold, $\mathcal{O}$ recovers $\widehat{v}$ from $\widehat{\boldsymbol{w}}$ and returns $\widehat{v}$ to $\mathcal{A}$. Otherwise, $\mathcal{O}$ returns $\widehat{v}:=\perp$ to $\mathcal{A}$. We note that $\mathcal{O}$ does not extract the committed values of the Stage 2 commitments in such right sessions.

The expected running time of $H_{2}^{b}(n, z)$ is at most poly $\left(n^{\log n}\right) \cdot T_{i}(n)$ after the start of Stage 2 on the left.

${ }^{6}$ Since the running time of $\mathcal{A}$ and $\mathcal{O}$ is at most poly $(n) \cdot T_{i+1.5}(n) \ll T_{i+2}(n)$, the strong computational binding property of $\operatorname{CCACom}_{i+3}^{1: 1}$ guarantees that the Stage 1 commitment has at most one committed value except with negligible probability. 
Hybrid $H_{3}^{b}(n, z)$ is the same as $H_{2}^{b}(n, z)$ except that in Stage 2 on the left, the left committer commits to 0 instead of $s_{j}$ for each $j \notin \Gamma$.

Since $\mathcal{A}$ receives no information about $\left\{s_{j}\right\}_{j \notin \Gamma}$ on the left in $H_{3}^{0}(n, z)$ and $H_{3}^{1}(n, z)$, and since $s$ is $(n+1)$-out-of- $10 n$ secret sharing, the output of $H_{3}^{0}(n, z)$ and that of $H_{3}^{1}(n, z)$ are identically distributed. Then, we consider the following claims. In what follows, we use $\mathrm{H}_{i}^{b}(n, z)$ to denote the output of experiment $H_{i}^{b}(n, z)$.

Claim 4. For each $b \in\{0,1\},\left\{\mathrm{H}_{0}^{b}(n, z)\right\}_{n \in \mathbb{N}, z \in\{0,1\}^{*}}$ and $\left\{\mathrm{H}_{1}^{b}(n, z)\right\}_{n \in \mathbb{N}, z \in\{0,1\}^{*}}$ are computationally indistinguishable.

Claim 5. For each $b \in\{0,1\},\left\{\mathrm{H}_{1}^{b}(n, z)\right\}_{n \in \mathbb{N}, z \in\{0,1\}^{*}}$ and $\left\{\mathrm{H}_{2}^{b}(n, z)\right\}_{n \in \mathbb{N}, z \in\{0,1\}^{*}}$ are statistically indistinguishable.

Claim 6. For each $b \in\{0,1\},\left\{\mathrm{H}_{2}^{b}(n, z)\right\}_{n \in \mathbb{N}, z \in\{0,1\}^{*}}$ and $\left\{\mathrm{H}_{3}^{b}(n, z)\right\}_{n \in \mathbb{N}, z \in\{0,1\}^{*}}$ are computationally indistinguishable.

The proposition follows from these claims.

Proof (sketch of Claim 4). The view of $\mathcal{A}$ in $H_{0}^{b}(n, z)$ and that of $\mathcal{A}$ in $H_{1}^{b}(n, z)$ differ only in the committed values of $\mathrm{CECom}_{i+2}$ on the left. In addition, the running time of $H_{0}^{b}(n, z)$ and that of $H_{1}^{b}(n, z)$ are poly $(n) \cdot T_{i+1.5}(n) \ll T_{i+2}(n)$ (except for the brute force extraction of the Stage 1 commitment on the left in $\left.H_{1}^{b}(n, z)\right)$. Thus, by considering $\Gamma$ as non-uniform advice, we can prove indistinguishability from the hiding property of $\mathrm{CECom}_{i+2}$.

Next, we consider Claim [5] As in Section 4.2, we first show that in every right session of $H_{1}^{b}(n, z)$, the committed values of the Stage 2 commitments and those of Stage 3 commitments are 0.9-close. Formally, for any right session, let $\boldsymbol{s}^{(2)}=\left(s_{1}^{(2)}, \ldots, s_{10 n}^{(2)}\right)$ be the committed values of the Stage 2 commitments (if the committed value of the $j$-th commitment is not uniquely determined, $s_{j}^{(2)}$ is defined to be $\perp)$ and let $\boldsymbol{s}^{(3)}=\left(s_{1}^{(3)}, \ldots, s_{10 n}^{(3)}\right)$ be the committed values of the Stage 3 commitments. Then, for every $j \in[10 n]$, we say that the $j$-th column of this session is bad if $s_{j}^{(2)}=\perp, s_{j}^{(3)}=\perp$, or $s_{j}^{(2)} \neq s_{j}^{(3)}$. In addition, we say that $\mathcal{A}$ cheats in this session if the session is accepted and the number of bad columns is at least $n$. Then, we prove the following subclaim.

Subclaim 3. In any right session of $H_{1}^{b}(n, z), \mathcal{A}$ cheats with at most negligible probability.

Proof (sketch). At first sight, it seems that we can prove this subclaim by simply using the hiding property of CCACom ${ }_{i+3}^{1: 1}$ and the property of cut-and-choose technique (i.e., it seems that, since the committed value $\Gamma$ of the Stage 1 commitment on the right is hidden from $\mathcal{A}$, the probability that there are at least $n$ bad columns but the session is accepted is negligible). However, $\mathcal{A}$ interacts with the left committer as well as with $\mathcal{O}$, and the left committer "cheats" in 
the left session (i.e., on the left, the committed values of the Stage 2 commitments and those of the Stage 3 commitments are not 0.9 -close). Thus, $\mathcal{A}$ may be able to cheat in a right session by using the messages received on the left. A key to prove this subclaim is that the left session can be simulated by using the committedvalue oracle of $\mathrm{CCACom}_{i+3}^{1: 1}$ (i.e., if we know the committed value $\Gamma$ of the Stage 1 commitment on the left, we can simulate the later stages in polynomial time). Thus, the one-one CCA security of CCACom ${ }_{i+3}^{1: 1}$ guarantees that $\mathcal{A}$ cannot break the hiding property of CCACom C $_{i+3}^{1: 1}$ even with the messages of the left session. We can therefore use the cut-and-choose technique to prove the subclaim.

Given Subclaim [3, we can prove Claim 5 in a similar manner to Claim 2 in Section 4.2

Finally, we prove Claim 6.

Proof (sketch of Claim [6). $H_{2}^{b}(n, z)$ and $H_{3}^{b}(n, z)$ differ only in the committed values of $\mathrm{Com}_{i+1}$. Since the running time of $H_{2}^{b}(n, z)$ and that of $H_{3}^{b}(n, z)$ are poly $\left(n^{\log n}\right) \cdot T_{i}(n) \ll T_{i+1}(n)$ after the start of Stage 2 on the left, we can prove Claim 6 from the hiding property of $\mathrm{Com}_{i+1}$ (by considering $\Gamma$ of the left session and the answers of $\mathcal{O}$ for some right sessions as non-uniform advice). Here, we use the fact that $\mathrm{Com}_{i+1}$ is a 2-round commitment scheme. This fact enables us to rewind $\mathcal{A}$ in the right sessions of $H_{2}^{b}(n, z)$ without breaking the hiding property of $\operatorname{Com}_{i+1}$.

\section{One-One CCA Security for Long Tags from CCA Security for Short Tags}

In this section, we consider a transformation from any CCA-secure commitment scheme for tags of length $t(n)$ to a one-one CCA-secure commitment scheme for tags of length $2^{t(n)-1}$. The transformation are essentially the same as those in 24], which shows a transformation from any concurrent NM commitment scheme for short tags to a NM commitment scheme for long tags.

Lemma 3. Let $\epsilon<1$ be a positive constant, and assume the existence of oneway functions that are secure against $2^{n^{\epsilon}}$-time adversaries. Let $r(\cdot)$ and $t(\cdot)$ be arbitrary functions such that $t(n) \leq O(\log n)$, let $T_{i}(n) \stackrel{\text { def }}{=} 2^{(\log n)^{(2 / \epsilon)^{10 i+1}}}$ for $i \in \mathbb{N}$, and let $\mathrm{CCACom}_{i+1}$ be an $r(n)$-round commitment scheme that satisfies the following for any $T_{i+1}(n)$-time adversary.

- Statistical binding property, and

- CCA security for tags of length $t(n)$.

Then, there exists an $r(n)$-round commitment scheme $\mathrm{CCACom}_{i}^{1: 1}$ that satisfies the following for any $T_{i}(n)$-time adversary.

- Statistical binding property, and

- One-one CCA security for tags of length $2^{t(n)-1}$. 
If $\mathrm{CCACom}_{i+1}$ uses the underlying one-way function only in a black-box way, then $\mathrm{CCACom}_{i}^{1: 1}$ uses the underlying one-way function only in a black-box way.

Due to lack of space, the proof is deferred to the full version.

\section{Constant-Round Black-Box Composable Protocol}

In this section, we show a constant-round black-box construction of a general MPC protocol that satisfies angel-based UC security. Roughly speaking, the framework of angel-based UC security (called $\mathcal{H}$-EUC framework) is the same as the UC framework except that both the adversary and the environment in the real and the ideal worlds have access to a super-polynomial-time angel $\mathcal{H}$.

To construct our protocol, we use the following theorem, which we obtain by combining Lemmas 1, 2, and 3.

Theorem 1. Assume the existence of one-way functions that are secure against sub-exponential-time adversaries. Then, for any constant $\kappa \in \mathbb{N}$, there exists a constant-round commitment scheme that is CCA secure and $\kappa$-PQT-robust. This commitment scheme uses the underlying one-way functions only in a black-box way.

We additionally use the following results of [7] and [23].

Let $\langle C, R\rangle$ be any $r_{c c a}(n)$-round commitment scheme that is CCA secure and $\kappa$-robust for any constant $\kappa,\langle S, R\rangle$ be any $r_{o t}(n)$-round semi-honest $\mathrm{OT}$ protocol, and $\mathcal{H}$ be an angel that breaks $\langle C, R\rangle$ essentially in the same way as the committed-value oracle of $\langle C, R\rangle$ does. Then, Lin and Pass 23 showed that there exists a black-box $O\left(\max \left(r_{o t}(n), r_{c c a}(n)\right)\right)$-round protocol that securely realizes the ideal OT functionality $\mathcal{F}_{O T}$ in the $\mathcal{H}$-EUC framework. By using essentially the same security proof as that of [23], we can show that even when $\langle C, R\rangle$ is CCA secure and only $\kappa$-PQT-robust for a sufficiently large $\kappa$, the protocol of [23] is still secure if $\langle S, R\rangle$ is secure against any PQT adversary 7 Thus, we have the following theorem from [23].

Theorem 2. Assume the existence of an $r_{c c a}(n)$-round commitment scheme $\langle C, R\rangle$ that is CCA secure and $\kappa$-PQT-robust for a sufficiently large $\kappa$, and assume the existence of an $r_{\text {ot }}(n)$-round semi-honest oblivious transfer protocol $\langle S, R\rangle$ that is secure against any $\mathrm{PQT}$ adversary. Then, there exists an $O\left(\max \left(r_{c c a}(n), r_{o t}(n)\right)\right)$-round protocol that $\mathcal{H}$-EUC-realizes $\mathcal{F}_{O T}$. This protocol uses $\langle C, R\rangle$ and $\langle S, R\rangle$ only in a black-box way.

In [7, Canetti et al. showed the following.

Theorem 3 ( [7]). For every well-formed functionality $\mathcal{F}$, there exists a constantround $\mathcal{F}_{O T}$-hybrid protocol that $\mathcal{H}$-EUC-realizes $\mathcal{F}$.

Then, by combining Theorems 1, 2] and 3, we obtain the following theorem.

\footnotetext{
7 This is because $\kappa$-PQT-robustness guarantees that the committed-value oracle is useless in attacking any $\kappa$-round protocol if the protocol is PQT-secure.
} 
Theorem 4. Assume the existence of one-way functions that are secure against sub-exponential-time adversaries and constant-round semi-honest oblivious transfer protocols that are secure against quasi-polynomial-time adversaries. Then, there exists an angel $\mathcal{H}$ such that for every well-formed functionality $\mathcal{F}$, there exists a constant-round protocol that $\mathcal{H}$-EUC-realizes $\mathcal{F}$. This protocol uses the underlying one-way functions and oblivious transfer protocols only in a black-box way.

\section{References}

1. Barak, B., Sahai, A.: How to play almost any mental game over the net-concurrent composition via super-polynomial simulation. In: FOCS, pp. 543-552 (2005)

2. Canetti, R.: Universally composable security: A new paradigm for cryptographic protocols. In: FOCS, pp. 136-145 (2001)

3. Canetti, R., Fischlin, M.: Universally composable commitments. In: Kilian, J. (ed.) CRYPTO 2001. LNCS, vol. 2139, pp. 19-40. Springer, Heidelberg (2001)

4. Canetti, R., Goldreich, O., Goldwasser, S., Micali, S.: Resettable zero-knowledge. In: STOC, pp. 235-244 (2000)

5. Canetti, R., Kilian, J., Petrank, E., Rosen, A.: Black-box concurrent zeroknowledge requires (almost) logarithmically many rounds. SIAM J. Comput. 32(1), 1-47 (2002)

6. Canetti, R., Kushilevitz, E., Lindell, Y.: On the limitations of universally composable two-party computation without set-up assumptions. In: Biham, E. (ed.) EUROCRYPT 2003. LNCS, vol. 2656, pp. 68-86. Springer, Heidelberg (2003)

7. Canetti, R., Lin, H., Pass, R.: Adaptive hardness and composable security in the plain model from standard assumptions. In: FOCS, pp. 541-550 (2010)

8. Canetti, R., Lindell, Y., Ostrovsky, R., Sahai, A.: Universally composable two-party and multi-party secure computation. In: STOC, pp. 494-503 (2002)

9. Choi, S.G., Dachman-Soled, D., Malkin, T., Wee, H.: Black-box construction of a non-malleable encryption scheme from any semantically secure one. In: Canetti, R. (ed.) TCC 2008. LNCS, vol. 4948, pp. 427-444. Springer, Heidelberg (2008)

10. Choi, S.G., Dachman-Soled, D., Malkin, T., Wee, H.: Simple, black-box constructions of adaptively secure protocols. In: Reingold, O. (ed.) TCC 2009. LNCS, vol. 5444, pp. 387-402. Springer, Heidelberg (2009)

11. Dolev, D., Dwork, C., Naor, M.: Nonmalleable cryptography. SIAM J. Comput. 30(2), 391-437 (2000)

12. Garg, S., Goyal, V., Jain, A., Sahai, A.: Concurrently secure computation in constant rounds. In: Pointcheval, D., Johansson, T. (eds.) EUROCRYPT 2012. LNCS, vol. 7237, pp. 99-116. Springer, Heidelberg (2012)

13. Gentry, C., Wichs, D.: Separating succinct non-interactive arguments from all falsifiable assumptions. In: STOC, pp. 99-108 (2011)

14. Goldreich, O., Micali, S., Wigderson, A.: How to play any mental game or a completeness theorem for protocols with honest majority. In: STOC. pp. 218-229 (1987)

15. Goyal, V.: Constant round non-malleable protocols using one way functions. In: STOC, pp. 695-704 (2011)

16. Goyal, V.: Non-black-box simulation in the fully concurrent setting. In: STOC, pp. 221-230 (2013)

17. Goyal, V., Lin, H., Pandey, O., Pass, R., Sahai, A.: Round-efficient concurrently composable secure computation via a robust extraction lemma. Cryptology ePrint Archive, Report 2012/652 (2012), http://eprint.iacr.org/ 
18. Haitner, I.: Semi-honest to malicious oblivious transfer - the black-box way. In: Canetti, R. (ed.) TCC 2008. LNCS, vol. 4948, pp. 412-426. Springer, Heidelberg (2008)

19. Haitner, I., Ishai, Y., Kushilevitz, E., Lindell, Y., Petrank, E.: Black-box constructions of protocols for secure computation. SIAM J. Comput. 40(2), 225-266 (2011)

20. Ishai, Y., Kushilevitz, E., Lindell, Y., Petrank, E.: Black-box constructions for secure computation. In: STOC, pp. 99-108 (2006)

21. Lin, H.: Concurrent Security. Ph.D. thesis, Cornell University (2011)

22. Lin, H., Pass, R.: Non-malleability amplification. In: STOC, pp. 189-198 (2009)

23. Lin, H., Pass, R.: Black-box constructions of composable protocols without setup. In: Safavi-Naini, R., Canetti, R. (eds.) CRYPTO 2012. LNCS, vol. 7417, pp. 461-478. Springer, Heidelberg (2012)

24. Lin, H., Pass, R., Venkitasubramaniam, M.: Concurrent non-malleable commitments from any one-way function. In: Canetti, R. (ed.) TCC 2008. LNCS, vol. 4948, pp. 571-588. Springer, Heidelberg (2008)

25. Malkin, T., Moriarty, R., Yakovenko, N.: Generalized environmental security from number theoretic assumptions. In: Halevi, S., Rabin, T. (eds.) TCC 2006. LNCS, vol. 3876, pp. 343-359. Springer, Heidelberg (2006)

26. Micciancio, D., Ong, S.J., Sahai, A., Vadhan, S.: Concurrent zero knowledge without complexity assumptions. In: Halevi, S., Rabin, T. (eds.) TCC 2006. LNCS, vol. 3876, pp. 1-20. Springer, Heidelberg (2006)

27. Naor, M.: Bit commitment using pseudorandomness. J. Cryptology 4(2), 151-158 (1991)

28. Naor, M.: On cryptographic assumptions and challenges. In: Boneh, D. (ed.) CRYPTO 2003. LNCS, vol. 2729, pp. 96-109. Springer, Heidelberg (2003)

29. Pass, R.: Simulation in quasi-polynomial time, and its application to protocol composition. In: Biham, E. (ed.) EUROCRYPT 2003. LNCS, vol. 2656, pp. 160-176. Springer, Heidelberg (2003)

30. Pass, R.: Bounded-concurrent secure multi-party computation with a dishonest majority. In: STOC, pp. 232-241 (2004)

31. Pass, R., Lin, H., Venkitasubramaniam, M.: A unified framework for UC from only OT. In: Wang, X., Sako, K. (eds.) ASIACRYPT 2012. LNCS, vol. 7658, pp. 699-717. Springer, Heidelberg (2012)

32. Pass, R., Venkitasubramaniam, M.: On constant-round concurrent zero-knowledge. In: Canetti, R. (ed.) TCC 2008. LNCS, vol. 4948, pp. 553-570. Springer, Heidelberg (2008)

33. Pass, R., Wee, H.: Black-box constructions of two-party protocols from one-way functions. In: Reingold, O. (ed.) TCC 2009. LNCS, vol. 5444, pp. 403-418. Springer, Heidelberg (2009)

34. Pass, R., Wee, H.: Constant-round non-malleable commitments from subexponential one-way functions. In: Gilbert, H. (ed.) EUROCRYPT 2010. LNCS, vol. 6110, pp. 638-655. Springer, Heidelberg (2010)

35. Prabhakaran, M., Rosen, A., Sahai, A.: Concurrent zero knowledge with logarithmic round-complexity. In: FOCS, pp. 366-375 (2002)

36. Prabhakaran, M., Sahai, A.: New notions of security: Achieving aniversal composability without trusted setup. In: STOC, pp. 242-251 (2004)

37. Wee, H.: Black-box, round-efficient secure computation via non-malleability amplification. In: FOCS, pp. 531-540 (2010) 